\author{
Research Article \\ www.ijrap.net
}

\title{
MANAGEMENT OF KITIBHA KUSHTA (PSORIASIS): A CASE STUDY
}

NR Navoday Raju *1, Puripanda S Kamalakar ${ }^{2}$, S Deepika ${ }^{1}$, Tripathy TB ${ }^{3}$

${ }^{1}$ PG Scholar, Department of Kriya Shareera, SDM College of Ayurveda \& Hospital, Hassan, Karnataka, India

${ }^{2} \mathrm{PG}$ Scholar, Department of Samhita, SDM College of Ayurveda \& Hospital, Hassan, Karnataka, India

${ }^{3}$ Professor, Department of Swasthavrutta, SDM College of Ayurveda \& Hospital, Hassan, Karnataka, India

Received on: 15/07/16 Revised on: 10/12/16 Accepted on: 10/01/17

\author{
*Corresponding author \\ E-mail: drnrnraju@gmail.com
}

DOI: 10.7897/2277-4343.08115

\section{ABSTRACT}

A 34-years old male patient who was apparently healthy before 5 years, developed with rough skin lesions over scalp, upper and lower limbs associated with itching and powdery discharge which spread all over the body consulted our out- patient department. He was found to be nondiabetic and non- hypertensive. He was having habits like regular day sleep, intake of curd in night time, intake of non-vegetarian food and consumption of alcohol, oily and junk food. He consulted Siddha and Homeopathic physicians before but didn't get relief from the symptoms. He was diagnosed to be suffering with Kitibha Kushta vis-à-vis Psoriasis clinically. He was under our Ayurvedic management for the past two years and very satisfied with the results. One month back he suffered with Dengue fever and took allopathic medications which include anti-biotic and analgesics and there after aggressive relapse of the previous symptoms was seen which lead to the manifestation of depression in the patient. In this paper, successful management of this case with Shodhana and Shamana modalities is being highlighted which will be a documented evidence for the future case studies.

Keywords: Kitibha, Kushta, Psoriasis, Kandu, Shyavam, Khara Sparsha, Ughra Kandu.

\section{INTRODUCTION}

Kitibha Kushta manifests due to the aggravation of Tridosha especially dominance of Vata and Kapha. Mithyahara and Vihara vitiate Tridosha which further lead to the affliction and aggravation of Rasa, Rakta, Mamsa and Laseeka. Predominance of Vata can be elicited with symptoms like blackish discoloration, hardness, dryness and roughness to touch. Kapha Predominance can be appreciated with the presence of severe itching as a cardinal symptom.

Vitiation of Tridosha followed by affliction of four entities viz., Twak, Rakta, Mamsa and Laseeka leads to Kushta. ${ }^{1}$ These seven are called as the seven morbid factors (Sapta Dravya Sangraha) of Kushta. ${ }^{2}$ No Kushta manifests itself due to the aggravation of only one Dosha.

Kitibha is a type of Kushta (Kshudra Kushta) characterised by skin which is blackish brown in colour (Shyavata), rough in touch like a scar tissue (Kharatwam) and hard to touch (Parushatwam). ${ }^{3}$ Dry rough (Rukshata) and hard black skin with itching creating sound on scratching is seen in Kitibha. ${ }^{4} \mathrm{Kushta}$ characterised with discharging (Sraavi), round (Vruttam), dense (Ghanam), severely itching (Ughra Kandu) and oily black (Snigdha Krishna) skin is Kitibha. ${ }^{5}$

Ayurvedic management of this ailment with Shamana and Shodhana modalities is being discussed here under.

\section{MATERIALS AND METHODS}

Review of literature of concepts related to Kitibha Kushta and Psoriasis, interpretation of the symptoms along with successful Ayurvedic management of a patient. Present study was carried out in accordance with ethical principles by following International Conference of Harmonization-Good Clinical Practices Guidelines (ICH-GCP).

\section{Presenting Concern}

A 34-years old married male patient who was apparently healthy before 5 years, developed with skin lesions over scalp, upper and lower limbs associated with itching and powdery discharge which spread all over the body. He is a software professional belonging to middle class socio- economic Hindu family background. He consulted Siddha and Homeopathy physicians before but didn't get relief from the symptoms. He consulted our out- patient department for better results. He was found to be non- diabetic and non- hypertensive. He was having habits like regular day sleep, intake of curd tonight, intake of nonvegetarian food and consumption of alcohol, oily and junk food. He was diagnosed to be suffering with Kitibha Kushta vis-à-vis Psoriasis. He was under our Ayurvedic management for the past two years and very satisfied with the results. One month back he suffered with Dengue fever and took allopathic medications which include anti-biotic and analgesics and there after aggressive relapse of the previous symptoms was seen which lead to the manifestation of depression in the patient.

\section{Clinical Findings}

No similar family history was found. Patient was very much depressed with the symptomatic manifestations. On examination, the skin lesions were blackish brown spread onto scalp, abdomen, back, upper and lower limbs with powdery discharge. Lesions were dry, rough and hard to touch. Candle grease sign was positive. 
Table 1: Kitibha Kushta Features

\begin{tabular}{|c|c|}
\hline Shyavam/ Snigdha Krishna & Its meaning is blackish/ dark discoloration. It is because of vitiated Vata Dosha. \\
\hline Kharatwam & It conveys the sense of roughness to touch and is because of Vata Vitiation. \\
\hline Parushatwam & Hardness to touch. It is also because of Vata Dosha. \\
\hline Rukshatwam & Dryness of the skin seen in Kitibha because of vitiation of Vata Dosha. \\
\hline UghraKandu & Kandu is produced by the vitiated Kapha Dosha. Severe itching was seen in the patient. \\
\hline
\end{tabular}

Pathogenesis

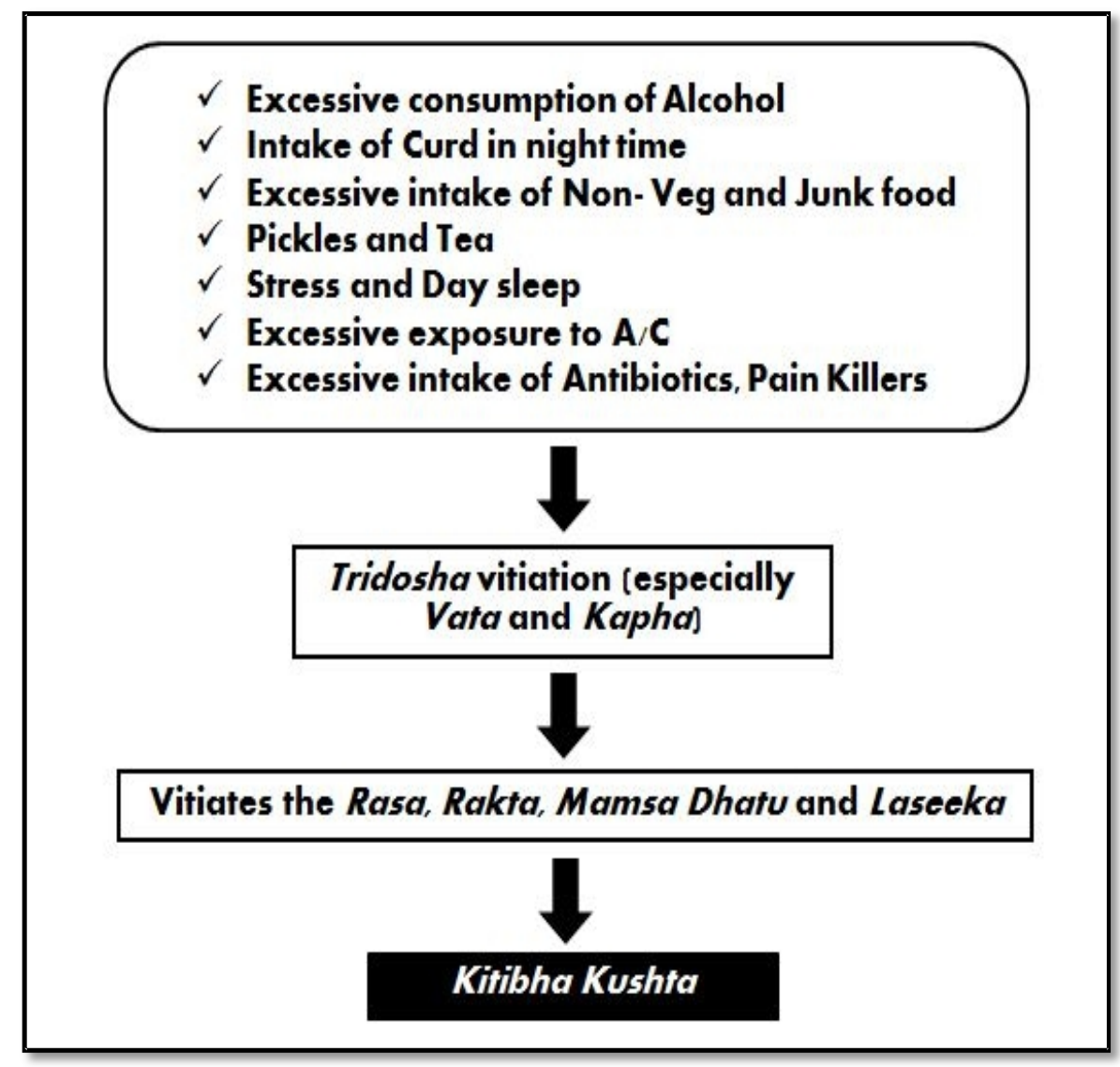

Figure 1: Pathogenesis of Kitibha Kushta

Diagnostic Focus and Assessment

Table 2: Comparison of Kitibha Kushta and Psoriasis

\begin{tabular}{|c|c|}
\hline Kitibha & Psoriasis $^{\mathbf{6}}$ \\
\hline Shyavam/ Snigdha Krishna & Erythematous lesions turn to black in chronic cases \\
\hline Kharatwam & Candle grease sign- Positive \\
\hline Parushatwam & Abnormal hardening seen in chronic cases \\
\hline Rukshatwam & Scales rough to touch \\
\hline Ughra Kandu & Severe itching observed \\
\hline
\end{tabular}

Therapeutic Focus and Assessment

Treatment Principle

Step 1: Manasika Chikitsa along with Deepana Pachana, Nitya Mrudu Virechana and Bahirparimarjana

Step 2: Sadyo Virechana

Step 3: Shodhana Chikitsa (Virechana) along with Samsarjana Krama

Step 4: Rasayana Prayoga along with Shamana Oushadhi (Abhyantara and Bahya) 


\section{Table 3: Treatment Schedule}

\begin{tabular}{|c|c|}
\hline Date & Medicines \\
\hline $\begin{array}{l}25-12-15 \\
\text { To } \\
28-12-15\end{array}$ & $\begin{array}{l}\text { Deepana Pachana: } \\
\text { Shiva Gulika } 2 \text { tablets morning and evening before food with Luke warm water } \\
\text { Nitya Mrudu Virechana: } \\
\text { Amalaki Churna 1/2 Teaspoonful morning and evening after food with Luke warm water } \\
\text { Manasika Chikitsa: } \\
\text { ManasamitraVati } 2 \text { tablets at night time after food } \\
\text { Taila Dhara with Ksheerabala Taila } \\
\text { Bahirparimarjana Chikitsa: } \\
\text { Nalpamaradi Taila for external application and Ushna Jala Snana } \\
\text { Sorion Ointment for external application after bath } \\
\text { Psorolin Soap for bathing }\end{array}$ \\
\hline $29-12-15$ & $\begin{array}{l}\text { Sadyo Virechana: } \\
\text { Manibhadra Guda40 grams with Luke warm water morning time (Virechana Vega-12) }\end{array}$ \\
\hline $30-12-15$ & $\begin{array}{l}\text { Shodhana Chikitsa: } \\
\text { Sneha pana with Kalyanaka Ghrita } 30 \mathrm{ml} \\
\text { Bahirparimarjana Chikitsa }\end{array}$ \\
\hline $31-12-15$ & $\begin{array}{l}\text { Shodhana Chikitsa: } \\
\text { Sneha pana with Kalyanaka Ghrita } 60 \mathrm{ml} \\
\text { Bahirparimarjana Chikitsa }\end{array}$ \\
\hline 01-01-16 & $\begin{array}{l}\text { Shodhana Chikitsa: } \\
\text { Sneha pana with Kalyanaka Ghrita } 90 \mathrm{ml} \\
\text { Bahirparimarjana Chikitsa }\end{array}$ \\
\hline $02-01-16$ & $\begin{array}{l}\text { Shodhana Chikitsa: } \\
\text { Sneha pana with Kalyanaka Ghrita } 110 \mathrm{ml} \\
\text { Snigdha Varchas observed } \\
\text { Bahirparimarjana Chikitsa }\end{array}$ \\
\hline $03-01-16$ to $05-01-16$ & Abhyanga with Nalpamaradi Taila and Mrudu Bhaspa Sweda \\
\hline $05-01-16$ & $\begin{array}{l}\text { Virechana: } \\
\text { Trivruth Lehya ( } 80 \text { grams) + Triphala Kashaya }(150 \mathrm{ml})+\text { Honey (Quantity sufficient) } \\
\text { Number of Vega- } 12 \text { and Samsarjana Krama advised. }\end{array}$ \\
\hline $06-01-16$ & $\begin{array}{l}\text { Patient was discharged tonight with following discharge medicines: } \\
\text { Rasayana Prayoga: } \\
\text { Shiva Gulika } 1 \text { tablet in the morning time } 1 \text { hour before food } \\
\text { Shamana Oushadhi (Abhyantara): } \\
\text { Arogyavardhini Vati } 2 \text { tablets morning and evening after food } \\
\text { Mahamanjishtadi Kashaya } 4 \text { teaspoonful morning and evening with equal water before food } \\
\text { Manasamitra Vati } 2 \text { tablets before going to bed } \\
\text { Nitya Virechana: } \\
\text { Manibhadra Guda } 1 / 2 \text { teaspoonful before going to bed } \\
\text { Shamana Oushadhi (Bahya): } \\
\text { Parantyadi Taila for external application before bath } \\
\text { Ointment Sorion for external application after bath } \\
\text { Psorolin Soap for bathing }\end{array}$ \\
\hline
\end{tabular}

Table 4: Outcome of the Treatment

\begin{tabular}{|c|c|c|}
\hline & Before treatment & After treatment \\
\hline Dark Blackish Discoloration (Shyavatwam) & Very much & No Roughness \\
\hline Roughness (Kharatwam) & Very Rough & No Hardness \\
\hline Hardness (Parushatwam) & Hardness found & No Dryness \\
\hline Dryness (Rukshatwam) & Very much & Reduced \\
\hline Itching (Kandu) & Very severe \\
\hline
\end{tabular}




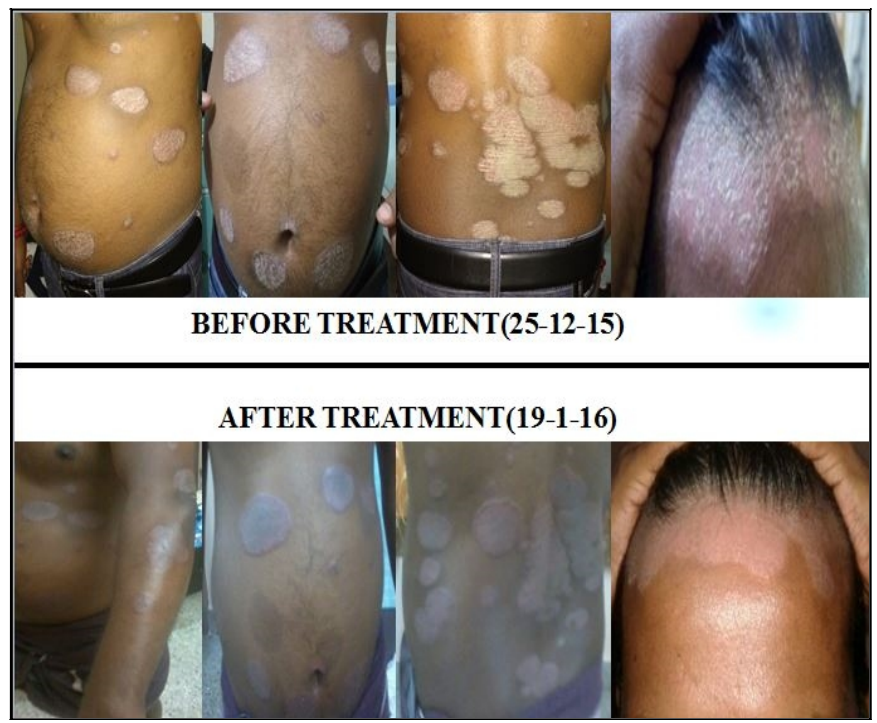

Figure 2: Before Treatment (BT) and After Treatment (AT)

\section{DISCUSSION}

Table 5: Prescribed Medications with Ingredients and their Actions

\begin{tabular}{|c|c|c|}
\hline Formulation & Ingredients & Actions \\
\hline $\begin{array}{c}\text { Ksheerabala Taila } \\
\text { (AFI, Ashtanga Hridaya Vatarakta Chikitsa) }\end{array}$ & Sida cordifolia, Ksheera, Sesamum indicum etc., & Vata Vyadhi, Balya etc. \\
\hline $\begin{array}{c}\text { Nalpamaradi Taila }^{8} \\
\text { (Sahasrayoga Tailaprakarana) }\end{array}$ & $\begin{array}{c}\text { Ficus benghalensis, Ficus racemosa } \\
\text { Ficus religiosa, Ficus virens, Terminalia chebula, } \\
\text { Phyllanthus emblica } \text { etc. }\end{array}$ & $\begin{array}{l}\text { Tvak Roga, Visarpa, Kushta, Paama, } \\
\text { Kandu, Pidaka etc. }\end{array}$ \\
\hline $\begin{array}{c}\text { Shiva Gulika9 } \\
\text { (Ashtanga Sangraha Vajeekarana) }\end{array}$ & $\begin{array}{c}\text { Asphaltum punjabianum, Alpinia galanga, Plumbago } \\
\text { zeylanica, Holarrhena antidysenterica, Triphala, } \\
\text { Azadirachta indica, Trichosanthes dioica etc. }\end{array}$ & Kushta, Pandu, Jwara etc. \\
\hline $\begin{array}{c}\text { Manasamitra Vati } \\
\text { (Sahasrayogam Gutika Prakarana) }\end{array}$ & $\begin{array}{c}\text { Sida cordifolia, Sida veronicaefolia, Aegle marmelos, } \\
\text { Convolvulus pluricaulis, Inula racemosa, Leptadeni } \\
\text { areticulata, Curcuma longa etc. }\end{array}$ & $\begin{array}{l}\text { Mano Dosha, Unmada, Apasmara, } \\
\text { Manda Buddhitwa etc. }\end{array}$ \\
\hline Sorion Topical & $\begin{array}{c}\text { Leptadeni areticulata, Rubia cordifolia, Curcuma } \\
\text { longa, Holarrhena antidysenterica, Cocos nucifera, } \\
\text { Hydnocarpus laurifolia, Cera alba }\end{array}$ & $\begin{array}{l}\text { Plaque, Palmoplantar, Scalp and other } \\
\text { Psoriasis, Cracked feet etc. }\end{array}$ \\
\hline Psorolin Soap & Wrightia tinctoria, Aloe vera & $\begin{array}{l}\text { All types of Psoriasis, Allergic skin } \\
\text { disorders, Seborrheic dermatitis, Dry } \\
\text { skin etc. }\end{array}$ \\
\hline $\begin{array}{c}\text { Manibhadra Guda } \\
\text { (Ashtanga Hridaya Kushta Chikitsa, AFI) }\end{array}$ & $\begin{array}{l}\text { Terminalia chebula, Phyllanthus emblica, Embelia } \\
\text { ribes, Operculina turpethum and Jaggery. }\end{array}$ & $\begin{array}{l}\text { Krimi, Bhagandara, Arshas and } \\
\text { Kushta etc. }\end{array}$ \\
\hline $\begin{array}{c}\text { Kalyanaka Ghrita }^{12} \\
\text { (Astanga Hridaya Uttarasthanam Unmaada } \\
\text { Prakarana, AFI) }\end{array}$ & $\begin{array}{c}\text { Terminalia chebula, Terminalia bellirica, } \\
\text { Phyllanthus emblica, Hemidesmus indicus, Haridra, } \\
\text { Berberis aristata, Rubia cordifolia } \text { etc. }\end{array}$ & $\begin{array}{l}\text { Apasmara, Bhutonmada, Kandu, } \\
\text { Daurbalya etc. }\end{array}$ \\
\hline 777 Oil & Wrightia tinctoria & All dry skin disorders \\
\hline Parantyadi Kera Taila & $\begin{array}{c}\text { Citrus limon, Jasminum grandiflorum, Picrorhiza } \\
\text { kurroa, Bacopa monnieri etc. }\end{array}$ & Different skin conditions \\
\hline $\begin{array}{c}\text { Arogyavardhini Vati }{ }^{13} \\
\text { (Rasaratna Samuchhayam) }\end{array}$ & $\begin{array}{l}\text { Picrorhiza kurroa, Hg, Sulphur, Loha (Fe) Bhasma, } \\
\text { Abhraka (Mica) Bhasma etc. }\end{array}$ & Skin diseases, Eczema, Sthoulya etc. \\
\hline $\begin{array}{c}\text { Mahamanjishtadi Kashaya }{ }^{\mathbf{1 4}} \\
\text { (Sarangadhara Madhyama Khanda) }\end{array}$ & $\begin{array}{l}\text { Rubia cordifolia, Cyperus rotundus, Picrorhiza } \\
\text { kurroa, Tinospora cordifolia, Saussurea lappa etc. }\end{array}$ & $\begin{array}{c}\text { Skin disorders, Scleroderma, } \\
\text { Psoriasis, Cellulitis, Furuncles etc. }\end{array}$ \\
\hline
\end{tabular}

The patient in this case was a Software Engineer with irregular food habits like consumption of excessive non-veg, curd (at night), regular fast food/ junk food. Because of professional stress and timings, he developed with sleep disturbances and habituated to untimely meals. The work area is a centralized air conditioned place which can vitiate the Dosha. Even though patient was found to take alcohol occasionally the other factors acted as triggers for the vitiation of Tridosha, which further afflicted Rasa, Rakta, Mamsa and Laseeka leading to the development of symptoms. Because of the carelessness of the patient, chronicity and actual recurrence nature of the disease it has become a challenge to manage the case. Even after successful management in the previous schedule of treatment this recent relapse was seen may be because of usage of medicines with side effects for Dengue fever from which he suffered.

Present treatment schedule has given him 60\% relief from the symptoms after Shodhana treatment i.e. Virechana and he was discharged in an improved condition with Shamana medicines. 
Table 6: Selection of Medicines

\begin{tabular}{|c|c|c|}
\hline Treatment/ formulation & Reason for selection & Probable mode of action \\
\hline Taila Dhara With Ksheerabala Taila & Sleeplessness and Depression & $\begin{array}{c}\text { As Dhara may cause dilatation of the micro vessels } \\
\text { allowing increased circulation to cerebrum }\end{array}$ \\
\hline Nalpamaradi Taila & Kandu, Rukshata, Shyavata & Twachyam, Kandughnam, Rakta Prasadana \\
\hline Shiva Gulika & Depression and Agni & Kandughna, Agni Deepana, Pachana, Rasayana \\
\hline Manasamitra Vati & Stress and Depression, Sleeplessness & $\begin{array}{c}\text { Induces sleep, Reduces stress, Medhyam, Indriya } \\
\text { Preenanam, Mano Doshaharam, Prana Anulomanam }\end{array}$ \\
\hline Amalaki Churna & Kandu, Sthoulya & Tridoshahara, Kandughna, Sthoulyahara, Malashodhanam \\
\hline $\begin{array}{c}\text { Sorion Ointment } \\
\text { Psorolin Soap }\end{array}$ & Mry Skin, Itching, Roughness, Hardness & Moisturization of skin \\
\hline $\begin{array}{c}\text { Manibhadra Guda } \\
\text { Kalyanaka Ghrita }\end{array}$ & Manasika and Twakgata Vikara & $\begin{array}{c}\text { Tridoshahara, Balya, Dhatupushtikara, Medhya (acts on } \\
\text { higher mental faculties) and Malanulomana etc., } \\
\text { Virechana }\end{array}$ \\
\hline $\begin{array}{c}\text { Parantyadi Kera Taila } \\
\text { (Vaidhyaratnam) }\end{array}$ & Dry Skin, Itching, Roughness, Hardness & Kandughnam, Kapha Vatahara \\
\hline Arogyavardhini Vati & Kandu & Kaphapittahara, Sramsana, Sthoulya \\
\hline Mahamanjishtadi Kashaya & Kandu, Rukshata, Kharatwa, Shyavata & Pittakapha Shamana, Rakta Prasadana, Kushtaghnam \\
\hline
\end{tabular}

\section{Future Direction}

Consideration of Psychological condition of the patient, status of Agni followed by repeated Shodhana and Rasayana drugs gives better results in all skin diseases.

\section{CONCLUSION}

This case study is a documented evidence for the successful management of Kitibha Kushtavis-à-vis Psoriasis through Shodhana and Shamana Chikitsa. This is one among the relapsing type of skin diseases so patient is advised to follow Pathyapathya like Ahara, Vihara, Achara and Vichara. Repeated Shodhana as per classics in accordance with Dosha, Kaala, Agni and Desha etc., should be administered to control the frequency of recurrence and further spread.

\section{REFERENCES}

1. Sharma RK, Dash B. English translation on Charaka Samhita of Agnivesha's. Reprint 2014.Chikitsa Sthana; Kushta Chikitsa: Chapter 7, Verse 7-8: Varanasi (India): Chowkhamba Sanskrit series office, 2014. P. 319-20.

2. Sharma RK, Dash B. English translation on Charaka Samhita of Agnivesha's. Reprint 2014.Chikitsa Sthana; Kushta Chikitsa: Chapter 7, Verse 9-10: Varanasi (India): Chowkhamba Sanskrit series office, 2014. P. 320-21.

3. Sharma RK, Dash B. English translation on Charaka Samhita of Agnivesha's. Reprint 2014.Chikitsa Sthana; Kushta Chikitsa: Chapter 7, Verse 22: Varanasi (India): Chowkhamba Sanskrit series office, 2014. P. 325.

4. Murthy KRS. Astanga Hrdayam. Reprint 2014. Nidana Sthana; Kushtaswitrakrumi Nidana: Chapter 14, Verse 21: Varanasi (India): Chowkhamba Krishnadas Academy, 2014. P. 139.

5. Sharma PV. Susruta Samhita. Reprint 2010. Nidana Sthana; Kushta Nidana: Chapter 5, Verse 14. Varanasi (India): Chaukhambha Visvabharati, 2010. P. 40.

6. Psoriasis [Online]. 2016 June [cited 2016 June 2]. Available from: https://en.wikipedia.org/wiki/Psoriasis.
7. Mahadevan L, Khan A, Gayathri R. Critical Analysis of Ayurvedic Formulations, $1^{\text {st }}$ ed. Kanyakumari (India): Sarada Mahadeva Iyer Ayurvedic Educational \& Charitable Trust; 2014. P. 407.

8. Mahadevan L, Khan A, Gayathri R. Critical Analysis of Ayurvedic Formulations, $1^{\text {st }}$ ed. Kanyakumari (India): Sarada Mahadeva Iyer Ayurvedic Educational \& Charitable Trust; 2014. P. 431.

9. Mahadevan L, Khan A, Gayathri R. Critical Analysis of Ayurvedic Formulations, $1^{\text {st }}$ ed. Kanyakumari (India): Sarada Mahadeva Iyer Ayurvedic Educational \& Charitable Trust; 2014. P. 302.

10. Mahadevan L, Khan A, Gayathri R. Critical Analysis of Ayurvedic Formulations, $1^{\text {st }}$ ed. Kanyakumari (India): Sarada Mahadeva Iyer Ayurvedic Educational \& Charitable Trust; 2014. P. 282.

11. Murthy KRS. Astanga Hrdayam. Reprint 2014. Chikitsa Sthana; Kushta Chikitsa: Chapter 19, Verse 31-32: Varanasi (India): Chowkhamba Krishnadas Academy, 2014. P. 477.

12. Mahadevan L, Khan A, Gayathri R. Critical Analysis of Ayurvedic Formulations, $1^{\text {st }}$ ed. Kanyakumari (India): Sarada Mahadeva Iyer Ayurvedic Educational \& Charitable Trust; 2014. P. 241.

13. Mahadevan L, Khan A, Gayathri R. Critical Analysis of Ayurvedic Formulations, $1^{\text {st }}$ ed. Kanyakumari (India): Sarada Mahadeva Iyer Ayurvedic Educational \& Charitable Trust; 2014. P. 306.

14. Mahadevan L, Khan A, Gayathri R. Critical Analysis of Ayurvedic Formulations, $1^{\text {st }}$ ed. Kanyakumari (India): Sarada Mahadeva Iyer Ayurvedic Educational \& Charitable Trust; 2014. P. 147.

\section{Cite this article as:}

NR Navoday Raju, Puripanda S Kamalakar, S Deepika, Tripathy TB. Management of Kitibha kushta (Psoriasis): A case study. Int. J. Res. Ayurveda Pharm. 2017;8(1):72-76 http://dx.doi.org/ 10.7897/2277-4343.08115

Disclaimer: IJRAP is solely owned by Moksha Publishing House - A non-profit publishing house, dedicated to publish quality research, while every effort has been taken to verify the accuracy of the content published in our Journal. IJRAP cannot accept any responsibility or liability for the site content and articles published. The views expressed in articles by our contributing authors are not necessarily those of IJRAP editor or editorial board members. 